Tohoku J. exp. Med, 1969, 97, 95-96

Short Report

\title{
Lidoflazine as an Antiarrhythmic Drug
}

\author{
Mitsuo Miyahara, Osamu limura, \\ Minoru Yokoyama and Koki Hoshikawa \\ The Second Department of Internal Medicine (Prof. M. Miyahara), \\ Sapporo Medical College, Sapporo
}

Since Schaper and his coworkers ${ }^{1-3}$ reported an excellent coronary vasodilating action of lidoflazine, a drug originally prepared in Janssen Pharmaceutica Institute, on conscious dogs with a previously implanted electromagnetic flowmeter, the antianginal effect of the drug has been clinically investigated in our own and other laboratories. Its usual dose is 90 or $120 \mathrm{mg}$ per day.

Recently, we administered a large dose of lidoflazine (90 $\mathrm{mg}$ three times a day) to a patient with atrial fibrillation due to coronary heart disease, and a reversion to sinus rhythm was unexpectedly recognized. Since there has been no report on such a defibrillating effect of this drug, and since a chemical structure of lidoflazine is similar to that of lidocain which has been recommended for clinical use as an effective antiarrhythmic drug, ${ }^{4-7}$ we think it adequate to evaluate the effect of lidoflazine in the treatment of atrial fibrillation and of other arrhythmias. The present paper is a preliminary report of the results obtained up to the present.

Patients with established atrial fibrillation were divided into two groups according to the procedure of medication. The first group consisted of seven patients who were given a large dose of lidoflazine (270 or $180 \mathrm{mg}$ per day). The second group consisted of five patients who received an initial dose of $60 \mathrm{mg}$ per $\mathrm{day}$, and then the dose was gradually increased by 30 or $60 \mathrm{mg}$ up to $270 \mathrm{mg}$ as the maximum dose. In addition, the drug was given to three other patients with paroxysmal atrial fibrillation and to another with paroxysmal tachycardia.

In all seven cases of the first group, established atrial fibrillation was converted to sinus rhythm. A reversion occurred within one to two days in 5 out of 7 patients, and within 4 to six days in the other two. Fibrillation did not reappear despite immediate discontinuation of the drug in all except two cases. In two cases of patients with mitral valvular disease in a state of congestive heart failure who were given a maintenance dose of digitoxin, several arrhythmias such as atrioventricular block, ventricular premature beat, ventricular tachycardia or ventricular fibrillation (in one case) occurred with only transient reversion to sinus rhythm. These arrhythmias occurred after one day (total $270 \mathrm{mg}$ ) in one case and after only $120 \mathrm{mg}$ ( $60 \mathrm{mg}$, two times) in the other.

Received for publication, September 19, 1968. 
In contrast to the first group, none of five patients in the second group responded to lidoflazine with improved cardiac rhythm, though the total dose was larger than that in the first. Moreover, the administration must be discontinued because of the appearance of tachycardia, frequent premature beat or conversion to atrial flutter, probably on account of the toxic effect of this drug.

A prevention of paroxysmal arrhythmia was definitively observed in one out of 3 cases of paroxysmal atrial fibrillation, and in another of paroxysmal tachycardia.

Our results show that lidoflazine has an excellent defibrillating effect when its initial dose is large enough, but toxic action may develop by its accumulation, under some conditions. A further investigation is in progress now to find out a more precise procedure of the administration, and about indication, toxicity, etc. of this drug.

\section{References}

1) Schaper, W.K.A., Xhonneux, R. \& Jageneau, A.H.M. Stimulation of the coronary collateral circulation by lidoflazine. Naunyn-Schmiedeberg's Arch.exp. Path. Pharmak., $1965,252,1-8$.

2) Schaper, W.K.A., Xhonneux, R., Jageneau, A.H.M. \& Janseen, P.A.J. The cardiovascular pharmacology of lidoflazine, a long-acting coronary vasodilator. J. Pharmacol. exp. Ther., 1966, 152, 265-274.

3) Jageneau, A.H.M. \& Schaper, W.K.A. The effectiveness of lidoflazine and other coronary vasodilators after oral administration in the trained non-anesthetized dog. Arzneimittelforsch., 1967, 17, 582-587.

4) Sonthworth, J.L., MeKusick, V.A., Peirce, E.C. \& Rawson, F.L. Ventricular fibrillation precipitated by cardiac catheterization. J. Ames. med. Ass., 1950, 143, 717-720.

5) Annotation: Management of acute myocardial infarction. Lancet, 1967, 2, 665.

6) Spracklen, F.H.N., Kimering, J.J., Besterman, E.M.M. \& Litchfeld, J.W. Use of lignocaine in treatment of cardiac arrhythmias. Brit. med. J., 1968, 1, 89-91.

7) Gianelly, R., von der Groebene, J.O., Spivack, A.P. \& Harrison, D.C. Effect of lidocaine on ventricular arrhythmias in patients with coronary heart disease. New Engl. J. Med., 1967, 277, 1215-1219. 